\title{
Ting Huang and Powder Diffraction Journal, PDJ
}

This issue of Powder Diffraction is dedicated in memory of Dr. Ting C. Huang for his enthusiasm and passion that he showed in working with this journal. Early in PDJ history, Ting served on the Editorial Advisory Board, as an International Reports Editor, Editor for New Diffraction Data, and Associate Editor-in-Chief, before he became Editor-in-Chief in 1999. From 1999 to 2013, Ting worked continuously to enhance the status and international outreach of PDJ. I asked Dr. Victor Buhrke, a friend of Ting and a friend of all of us in the X-ray Scattering Community, to write a dedication. On the following page you will find a tribute to Dr. Ting Huang, our colleague and our friend.

A search for a new Editor-in-Chief is underway. In the interim, Powder Diffraction will continue to receive, review and publish manuscripts under the guidance of Editors Xiaolong Chen, Jose Miguel Delgado, and Norberto Masciocchi, Editor for New Diffraction Data Soorya Kabekkodu, Editors for Crystallographic Education James
Kaduk and Brian Toby, International Reports Editor Winnie Wong-Ng, Calendar of Meetings and Workshops Editor Gang Wang, and Managing Editor Nicole Ernst Boris. All of us will be honored to continue and grow the legacy Ting has left for the materials community.

In that spirit, for 2013, supplemental issues of Powder Diffraction from proceedings of the European Powder Diffraction Conference (EPDIC 13), the 11th Chinese National Conference on X-ray Diffraction, and select papers from the Denver X-ray Conference proceedings Advances in $X$-ray Analysis, have been published in addition to the regular issues of Powder Diffraction; all with access for on line subscribers to PDJ. Look for more in 2014 including the proceedings from the Australian X-ray Analytical Association.

Tom Blanton Editor-in-Chief (interim) 